\title{
Other immunomodulatory agent-related lymphoproliferative diseases: a single-center series of 72 biopsy-confirmed cases
}

\author{
Lukas Marcelis $^{1}$ - Charlien Berghen ${ }^{2}$ - Alexandra De Zutter ${ }^{1}$ Pauline Biesemans ${ }^{1} \cdot$ Peter Vandenberghe $^{3}$. \\ Gregor Verhoef $^{3,4} \cdot$ Olivier Gheysens $^{5} \cdot$ Xavier Sagaert $^{1,6} \cdot$ Daan Dierickx $^{3} \cdot$ Thomas Tousseyn $^{1,6}$
}

Received: 19 January 2018 / Revised: 12 March 2018 / Accepted: 24 March 2018 / Published online: 15 May 2018

(c) United States \& Canadian Academy of Pathology 2018

\begin{abstract}
Ongoing development of new drugs, as well as novel indications in the treatment of autoimmune diseases leads to the increasing use of immunomodulatory and immunosuppressive drugs. Immunomodulatory agent-related lymphoproliferative disorders are a known and potentially life threatening complication of chronic administration of these drugs, but are less well characterized compared with post-transplant lymphoproliferative disorders. The heterogeneous drug targets, various underlying disease indications, different drug combinations used and relatively low incidence render data collection and interpretation difficult. In this retrospective paper, we describe the clinicopathological characteristics of a larger single-center series of 72 immunomodulatory agent-related lymphoproliferative disorder cases. We divided the cases according to the therapy, administered in the year preceding diagnosis of a lymphoproliferative disorder, in an immunosuppressive drug, an immunomodulatory drug and a combination of immunosuppressive and immunomodulatory drugs group. We observed differences in "time to lymphoproliferative disorder development" with a shorter time for all the immunomodulatory drugrelated cases combined (immunomodulatory and immunomodulatory + immunosuppressive $=$ immunomodulatory-all) vs immunosuppressive-only ( $p=0.0031)$. The proportion of malignant cases in patients receiving immunomodulatory therapy was, however, also significantly lower when compared with the immunosuppressive treated cases (43 vs $88 \% ; p=0.0184$ ). The immunomodulatory/suppressive agent-related lymphoproliferative disorders were less often associated with the Epstein-Barr virus (EBV) (31 vs 66\%; $p=1.829 \mathrm{e}-05)$ and the lymphoproliferative disorders incidence in the first year after immunomodulatory/immunosuppressive therapy initiation was lower (18 vs $41 \% ; p=0.04151$ )—compared with a published series of 140 post-transplant lymphoproliferative disorder cases from the same center. However, a similar histopathological spectrum from nondestructive, to polymorphic and monomorphic lesions as in post-transplant lymphoproliferative disorders is observed. With increasing use of immunosuppressive and especially immunomodulatory therapy, a higher incidence of immunomodulatory/suppressive agent-related lymphoproliferative disorders is to be expected. Life-long awareness for development of immunomodulatory/suppressive agent-related lymphoproliferative disorders with clinical follow-up and timely biopsies of suspicious lesions is required since these lymphoproliferative disorders arise both early after therapy initiation and many years later. Histopathological confirmation and correct classification is necessary to guide therapy and EBV ISH should be a part of routine pathological diagnostics.
\end{abstract}

These authors contributed equally: Lukas Marcelis, Charlien Berghen.

These authors jointly supervised this work: Daan Dierickx, Thomas Tousseyn.

Electronic supplementary material The online version of this article (https://doi.org/10.1038/s41379-018-0054-2) contains supplementary material, which is available to authorized users.

Thomas Tousseyn

thomas.tousseyn@uzleuven.be

Extended author information available on the last page of the article

\section{Introduction}

Development of lymphoproliferative disorders in patients is a known complication of chronic suppression or modulation of the immune system. This is best characterized in patients receiving immunosuppressive drugs post solid-organ or allogeneic stem cell transplantation. In this population, the life-long incidence of post-transplant lymphoproliferative disorders varies between 1 and $20 \%[1,2]$, depending on the type of graft and immunosuppressive drug regimen. The 2017 revised WHO classification of tumors of 
hematopoietic and lymphoid tissues [3] divides posttransplant lymphoproliferative disorders into four main subtypes: nondestructive, polymorphic, monomorphic, and classic Hodgkin lymphoma post-transplant lymphoproliferative disorder. Non-destructive post-transplant lymphoproliferative disorder, generally a polyclonal process, is characterized by preservation of the tissue architecture. Polymorphic post-transplant lymphoproliferative disorder consists of small to very large, Reed-Sternberg-like, B-cells in an abundant microenvironment composed of different immune cell types. The disorder is further characterized by destruction of the normal tissue architecture and frequently necrosis. Polymorphic post-transplant lymphoproliferative disorder can be either polyclonal or monoclonal, while monomorphic post-transplant lymphoproliferative disorder is generally monoclonal. The monomorphic and classic Hodgkin lymphoma types of post-transplant lymphoproliferative disorder are further categorized as in non-immunosuppressed patients, according to the lymphoma they resemble, most frequently diffuse large B-cell lymphoma. In the development of immunosuppressionrelated lymphoproliferative disorders, Epstein-Barr virus (EBV) is known to play a role and post-transplant lymphoproliferative disorders are frequently EBV driven [4].

Similar lymphoproliferative disorders have been recognized in patients with other indications for immunosuppression such as autoimmune disorder therapy and immunosuppression-related disorders such as primary immune disorders, HIV infection, and age-related immunesenescence. The first is related to a broad variety of drugs, either with immunomodulatory or immunosuppressive effect (Table 1). It is categorized in the 2017 revised WHO classification of tumors of hematopoietic and lymphoid tissues [5] as "other immunomodulatory-agent related lymphoproliferative diseases." Although, an increasing incidence is observed, the characterization of this group is still limited to small series (Suppl. Figure S1).

Research has tended to focus on lymphomas associated with either a single-specific autoimmune disease or with a specific drug, for example, rheumatoid arthritis and methotrexate-associated lymphoproliferative disorders [6-9], or a single lymphoma type, such as classical Hodgkin lymphoma [10-12], rather than the characteristics of the group of disorders as a whole. The series of 18 cases

Table 1 Classification of therapeutic agents

\begin{tabular}{|c|c|c|c|c|}
\hline Pharmaceutical (generic) & Class & Type & Main mechanism & \# Patients \\
\hline Ledertrexate (methotrexate) & IS & Antifolate & DNA synthesis inhibition & 34 \\
\hline Imuran (azathioprine) & IS & Purine analogue & DNA synthesis inhibition & 24 \\
\hline Sulfasalazine (salazopyrine) & IS & Aminosalicylate & Inconclusive $^{a}$ & 9 \\
\hline Plaquenil (hydrochloroquine) & IS & TLR blocker & TLR singalling inhibition & 8 \\
\hline Arava (leflunomide) & IS & DHODH inhibitor & DNA synthesis inhibition & 3 \\
\hline Neoral (cyclosporin) & IS & $\mathrm{CNI}$ & Inhibition of T-lymphocyte signaling & 2 \\
\hline Advagraf (tacrolimus) & IS & $\mathrm{CNI}$ & Inhibition of T-lymphocyte signalling & 2 \\
\hline Allochrysine (aurothiopropanolsulfonate) & IS & Gold containing Compound & Inhibition of reduction/oxidation (redox) enzymes & 2 \\
\hline Esbriet (pirfenidone) & IS & Small molecule & Broad antifibrotic and anti-inflammatory activities & 1 \\
\hline Mercaptopurine (purinethol) & IS & Purine analogue & Inhibits purine nucleotide synthesis & 1 \\
\hline Remicade (infliximab) & IM & Monoclonal antibody & Anti-TNF $\alpha$ & 14 \\
\hline Humira (adalimumab) & $\mathrm{IM}$ & Monoclonal antibody & Anti-TNF $\alpha$ & 9 \\
\hline Enbrel (etanercept) & $\mathrm{IM}$ & Monoclonal antibody & Anti-TNF $\alpha$ & 4 \\
\hline anti-TNF $\alpha$, not specified & $\mathrm{IM}$ & Monoclonal antibody & Anti-TNF $\alpha$ & 2 \\
\hline Roactemra (tocilizumab) & $\mathrm{IM}$ & Monoclonal antibody & Anti-IL6R & 2 \\
\hline Ilaris (canakinumab) & $\mathrm{IM}$ & Monoclonal antibody & Anti-IL1 $\beta$ & 2 \\
\hline Entyvio (vedolizumab) & $\mathrm{IM}$ & Monoclonal antibody & Anti-integrin $\alpha 4 / \beta 7$ & 1 \\
\hline Kineret (anakinra) & $\mathrm{IM}$ & Recombinant RA & Anti-IL1R & 1 \\
\hline Stelara (ustekinumab) & $\mathrm{IM}$ & Monoclonal antibody & Anti-IL12 and anti-IL23 & 1 \\
\hline Trocade & NA & Collagenase inhibitor & Selective inhibitor of matrix metalloproteinase- 1 & 1 \\
\hline
\end{tabular}

Table based on Kovarik et al. [14]; "\# Patients" is the number of patients who at one point received the specified pharmaceutical in the year before LPD diagnosis

$C N I$ calcineurin inhibitor, $D O D H$ dihydro-orotate dehydrogenase, IL interleukin, $I M$ immunomodulatory, $I S$ immunosuppressive, $L P D$ lymphoproliferative disorder, $N A$ not available, $R A$ receptor antagonist, $T L R$ toll-like receptor, $T N F$ tumor necrosis factor

${ }^{a}$ Proposed mechanisms are inhibition of cyclo-oxygenase and interleukin-1, TNF activation and scavenging of reactive oxygen species [72] 
published by Hasserjian et al. [13] in 2009 is, to the best of our knowledge, the only study to date that undertakes this broader approach. A possible etiological distinction can be made between immunosuppressive and immunomodulatory pharmaceuticals, as proposed previously [14] (Table 1). Immunosuppressive agents, such as methotrexate, inhibit immune cell proliferation, while immunomodulatory agents, for instance TNF $\alpha$ blockers, consist mainly of monoclonal antibodies and alter the immune response by targeting relevant cytokines. The exact link between immunomodulatory/immunosuppressive medication and lymphoproliferative disorders is still under discussion with data suggesting that the underlying autoimmune disease activity might be more important than the potential effects of therapy $[15,16]$. Epidemiological studies conducted to determine the role of a specific drug are difficult to perform due to the low amount of "hits" in the population [17]. For instance in inflammatory bowel disease (IBD) patients treated with thiopurines the reported increased risk to develop lymphoma varies from a 0 to a 31 -fold increase [18-25] and two published meta-analyses on the subject contradict each other [26, 27]. Determination of the impact of a specific therapeutic agent is further complicated by the parallel and/or successive administration of multiple drugs, potentially explaining why some studies did not find an increased risk for lymphoma in patients under immunomodulatory anti-TNF $\alpha$ therapy [17,28-31].

As new immunomodulatory agents continue to become available and the number of patients receiving immunomodulatory/suppressive therapy increases, more awareness for this entity is warranted. With this retrospective clinicopathological characterization of 72 cases of immunomodulatory/suppressive agent-related lymphoproliferative disorders diagnosed in our center, we aim to better describe this entity. By focusing on all relevant medications and underlying diseases rather than on a specific combination, the result should be a general overview of the disease and its characteristics. We aim to describe differences between immunomodulatory and immunosuppressive therapyrelated lymphoproliferative disorders and investigate the role of EBV in the pathogenesis.

\section{Materials and methods}

\section{Patient selection}

A list of biopsy reports over the last 19 years from the database at the Department of Pathology (University Hospitals KU Leuven, Belgium) was generated based upon the search terms ("lymphoma" OR "lymphoproliferation") AND ("autoimmune disorder" or "immunosuppressive drugs"). Patients who had undergone solid organ or hematopoietic stem cell transplantations and patients who received immunotherapy as treatment for lymphoma or other malignancies were removed from the list. One patient was being treated for polychondritis, while being HIV positive. Since the viral load was under control and CD4 + cell count was within normal range at the time of biopsy, this patient was not excluded. The intake of immunomodulatory/immunosuppressive medication at the time of the histological diagnosis was confirmed in the clinical records. Histology was reviewed by two expert hematopathologist (T.T. and X.S.) and cases were classified according to the 2017 revised WHO criteria [3]. In total 72 patients with biopsy-confirmed lymphoproliferative disorder were retained. The cases were then subdivided as either immunosuppressive therapy related, immunomodulatory therapy related or both immunosuppressive and immunomodulatory related, as based on a previously described classification of existing therapeutics [14]. Only the drug type administered in the 12 months prior to the lymphoproliferative disorder diagnosis was taken into consideration for subclassification. Only one case received no immunomodulatory/immunosuppressive medication in the year before diagnosis. This patient had received methotrexate up to 14 months prior to diagnosis and was included in the immunosuppressive therapy group. For patients where therapy was initiated before digital recordkeeping $(n=22)$ or who were referred by other medical centers $(n=25)$ exact treatment durations were often unclear, in these cases the date of the autoimmune disease diagnosis was used as the date of therapy initiation and we assumed continuous therapy unless explicitly stated otherwise.

This study was approved by the Ethical Committee of the University Hospitals Leuven (S-55498) and was conducted according to the Declaration of Helsinki.

\section{Clinical data}

Clinical data were retrieved from the clinical files available at UZ Leuven. The following characteristics were checked: age, sex, underlying disease, (sub)type of lymphoma or lymphoproliferative disorder, staging according to the Ann-Arbor staging system, the International Prognostic Index scoring, and when patients were younger than 60, age-adjusted International Prognostic Index scoring score was used. Extranodal disease, medication information (type and duration), as well as follow-up data were collected. Time to lymphoproliferative disorder development was calculated between the initiation of the autoimmune disease as well as the initiation of the immunosuppressive/ modulatory medication. Treatment response and outcome were assessed. 


\section{Immunohistochemistry and EBV-encoded RNA (EBER) in situ hybridization (ISH)}

Formalin-fixed paraffin-embedded sections were used for all stainings. Slides were immunostained automatically (DAKO, Carpenteria, CA, USA) according to manufacturers' protocol. All antibodies were purchased from DAKO unless stated otherwise (Supplemental list). Immunostains were performed on a case-by-case basis as required for establishing a diagnosis according to the 2017 Revised WHO classification. The presence of EBV was determined by EBER and performed as previously reported [32]. All EBER-positive biopsies with sufficient material were stained for EBV viral protein expression: latency membrane protein (LMP1), LMP2A, EBNA1, EBNA2 (Abcam, Cambridge Science Park, Boston, USA), and ZEBRA (BZ1). Based on the expression of Epstein-Barr viral LMP and nuclear antigen 2 (EBNA2), we defined three different conventional latency types of EBV infection. LMP1 -/EBNA2-, LMP1+/EBNA2-, and LMP1+/EBNA2+ represent latency types I, II, and III (respectively also referred to as restricted, intermediate and broad latency). Occurrence of lytic viral replication was determined by ZEBRA staining. The number of ZEBRA-positive cells was determined based on the range of ZEBRA-positive nuclei in 5 high power fields (HPFs) $(\times 400)$ as previously described [33].

\section{Clonality PCR}

Immunoglobulin heavy chain $(\operatorname{IgH})$, kappa light chain $(\operatorname{IgK})$ and T-cell receptor rearrangements were studied by multiplex PCR with BIOMED-2 primers using a BIOMED-2 PCR based protocol [34] as described in the supplemental data.

\section{Statistical analysis}

Statistical analysis was performed using R software (v3.4.3, session information in supplemental data). A significance threshold of 0.05 was assumed for all analyses. Overall survival was computed from the date of diagnosis until last follow-up or death of all causes. Overall survival curves were generated by Kaplan-Meier analysis and statistical significance $(p<0.05)$ was determined with the log-rank test. The cases were divided in groups based on the therapy for the underlying autoimmune disorder as described. Differences in proportions were tested using $\mathrm{Chi}^{2}$ tests without Yates correction and Fisher's exact test in case of small cell count $(n<5)$. The Shapiro-Wilk normality test was used to determine if data was normally distributed. Differences in means were tested using the Welch 2 sample $t$-test for normal distributed data and the Mann-Whitney $U$-test in the other cases. Since this is a retrospective study with a limited amount of cases, a correction for multiple testing was not applied and trends $(p \geq 0.05)$ are also reported. As the immunomodulatory and immunomodulatory + immunosuppressive group showed strong similarities in the evaluated characteristics, an additional analysis was done between no "immunomodulatory therapy" (group IS) vs "immunomodulatory therapy given" (IM-All = IM and IS + IM).

\section{Results}

\section{General characteristics}

Of the 72 retained patients (full list in supplementary table), 42 belonged to the immunosuppressive group, 10 to the immunomodulatory group and 20 to the immunosuppressive + immunomodulatory group (Table 2 ). Patients in the immunosuppressive group were significantly older than those in the immunomodulatory and immunosuppressive + immunomodulatory groups with an average age of 55.5 years vs 41.1 and 45.1 years, respectively. Rheumatoid arthritis and IBD represented 63\% (45/72) of patients. Underlying autoimmune diseases present in only 3 or fewer patients were psoriasis $(n=3)$, chronic autoimmune pancreatitis $(n=1)$, interstitial lung disease $(n=1)$, membranous glomerulonephritis $(n=1)$, myasthenia gravis $(n=1)$, relapsing polychondritis $(n=1)$, and sarcoidosis $(n=1)$. Ankylosing spondylitis and Graves' disease were each present in 1 patient but only in combination with other diseases listed before. The proportion of high International Prognostic Index scoring score and Ann-Arbor stage were compared between the different therapy groups. An International Prognostic Index scoring score of 2-4 and AnnArbor stage of III-IV were considered as high for this analysis. Significant differences were observed as indicated in bold (Table 2). The immunosuppressive group had an overall higher International Prognostic Index scoring score with a score of 2 or higher in 70\% (19/27) of cases compared with $0 \%(0 / 5)$ and 19\% (3/16) in immunomodulatory and immunomodulatory + immunosuppressive groups, respectively. Ann-Arbor staging revealed a similar trend with a high stage in $70 \%(21 / 30)$ of the immunosuppressive group compared with 50\% (3/6) and 44\% (7/16) in immunomodulatory and immunomodulatory + immunosuppressive groups, respectively. Lymphoproliferative disorder with extranodal involvement was present in $54 \%$ of patients (39/72) and 36\% (26/72) had only extranodal disease with no significant differences between groups.

\section{Histopathological and molecular features}

Cases were subdivided in non-destructive/reactive, polymorphic lymphoproliferative disorders, non-Hodgkin 
Table 2 General characteristics

\begin{tabular}{|c|c|c|c|c|c|c|c|c|}
\hline \# Patients & $\begin{array}{l}\text { IS } \\
\text { therapy } \\
42\end{array}$ & $\begin{array}{l}\text { IM } \\
\text { therapy } \\
10\end{array}$ & $\begin{array}{l}\mathrm{IM}+\mathrm{IS} \\
20\end{array}$ & $\begin{array}{l}\text { All cases } \\
72\end{array}$ & $\begin{array}{l}\text { IS vs IM } \\
p \text {-value }\end{array}$ & $\begin{array}{l}\text { IS vs IS + IM } \\
p \text {-value }\end{array}$ & $\begin{array}{l}\text { IM vs IS } \\
+ \text { IM } \\
p \text {-value }\end{array}$ & $\begin{array}{l}\text { IS vs IM-all } \\
p \text {-value }\end{array}$ \\
\hline Age, median (range), years & $\begin{array}{l}56.5 \\
(17-84)\end{array}$ & $\begin{array}{l}43.5 \\
(13-69)\end{array}$ & $\begin{array}{l}47.5 \\
(12-76)\end{array}$ & $\begin{array}{l}52 \\
(12-84)\end{array}$ & 0.0402 & 0.0371 & 0.5699 & 0.0067 \\
\hline Sex, men:women (ratio) & $\begin{array}{l}21: 21 \\
(1.00)\end{array}$ & $6: 4(1.5)$ & $\begin{array}{l}11: 8 \\
(1.38)\end{array}$ & $\begin{array}{l}38: 34 \\
(1.12)\end{array}$ & 0.7127 & 0.9232 & 1 & 0.5764 \\
\hline \multicolumn{9}{|c|}{ Underlying disease $(+$ when in combination) } \\
\hline Rheumatoid arthritis & $19(+1)$ & $1(+1)$ & $4(+1)$ & $22(+3)$ & & & & \\
\hline Crohn's disease & $9(+1)$ & 5 & $3(+1)$ & $17(+2)$ & & & & \\
\hline Ulcerative colitis & $5(+1)$ & 1 & $4(+1)$ & $10(+2)$ & & & & \\
\hline Juvenile idiopathic arthritis & 0 & 2 & 2 & 4 & & & & \\
\hline Sjögren's syndrome & $4(+1)$ & 0 & 0 & $4(+1)$ & & & & \\
\hline $\begin{array}{l}\text { Systemic lupus } \\
\text { erythematosus }\end{array}$ & 2 & 0 & $0(+2)$ & $2(+2)$ & & & & \\
\hline Other & 7 & 0 & 3 & 10 & & & & \\
\hline \multicolumn{9}{|l|}{ Classification } \\
\hline Non-Hodgkin lymphoma & 35 & 3 & 6 & 44 & $0.0184^{\mathrm{a}}$ & $5.9970 \mathrm{E}-05^{\mathrm{a}}$ & $1^{\mathrm{a}}$ & $2.943 E-05^{\mathrm{a}}$ \\
\hline Hodgkin lymphoma & 2 & 2 & 2 & 6 & & & & \\
\hline $\begin{array}{l}\text { Polymorphic } \\
\text { B-cell LPD }\end{array}$ & 3 & 2 & 3 & 8 & & & & \\
\hline Non-destructive LPD & 2 & 2 & 8 & 12 & & & & \\
\hline Unclassifiable & 0 & 1 & 1 & 2 & & & & \\
\hline \multicolumn{9}{|l|}{$I P I$} \\
\hline 0 & 3 & 1 & 6 & 10 & $0.0001^{b}$ & $0.0016^{b}$ & $0.5489^{\mathrm{b}}$ & $0.0005^{\mathrm{b}}$ \\
\hline 1 & 5 & 4 & 7 & 16 & & & & \\
\hline 2 & 9 & 0 & 1 & 10 & & & & \\
\hline 3 & 9 & 0 & 2 & 11 & & & & \\
\hline 4 & 1 & 0 & 0 & 1 & & & & \\
\hline NA & 15 & 5 & 4 & 24 & & & & \\
\hline \multicolumn{9}{|l|}{ Ann-Arbor stage } \\
\hline I & 7 & 1 & 6 & 13 & 0.1608 & 0.0823 & 1 & 0.0359 \\
\hline II & 3 & 2 & 3 & 8 & & & & \\
\hline III & 9 & 1 & 7 & 17 & & & & \\
\hline IV & 12 & 2 & 0 & 14 & & & & \\
\hline NA & 12 & 4 & 4 & 20 & & & & \\
\hline \multicolumn{9}{|l|}{ Extranodal disease } \\
\hline No & 18 & 4 & 11 & 33 & 1.0000 & 0.3704 & 0.6999 & 0.5487 \\
\hline Yes & 24 & 6 & 9 & 39 & & & & \\
\hline \multicolumn{9}{|l|}{ time until $L P D$} \\
\hline Median, months & 72.45 & 16.67 & 25.33 & 47.55 & & & & \\
\hline Mean, months & 94.55 & 27.97 & 44.68 & 72.11 & 0.3266 & 0.9274 & 0.1732 & 0.0031 \\
\hline$<1$ year & 4 & 1 & 5 & 10 & 1 & 0.2473 & 1 & 0.2943 \\
\hline$>1$ year & 33 & 6 & 14 & 53 & & & & \\
\hline NA & 5 & 3 & 0 & 9 & & & & \\
\hline
\end{tabular}

IM immunomodulatory, IPI International Prognostic Index, IS immunosuppressive, LPD lymphoproliferative disorder, NA not available ${ }^{a}$ Non-Hodgkin lymphoma vs non-destructive and polymorphic LPD

${ }^{\mathrm{b}}$ IPI Low (0-1) vs High (2-4).

${ }^{\mathrm{c}}$ Ann-Arbor stage Low (I-II) vs High (III-IV)

${ }^{\mathrm{d}}$ Significant values $(\mathrm{p}<0.05)$ are in bold. 
Table 3 EBV and molecular characteristics

\begin{tabular}{|c|c|c|c|c|}
\hline \# Patients & $\begin{array}{l}\text { IS therapy } \\
42\end{array}$ & $\begin{array}{l}\text { IM therapy } \\
10\end{array}$ & $\begin{array}{l}\text { IS + IM } \\
20\end{array}$ & $\begin{array}{l}\text { All cases } \\
72\end{array}$ \\
\hline \multicolumn{5}{|l|}{$E B V(E B E R I S H)$} \\
\hline Positive & 11 & 2 & 6 & 19 \\
\hline Negative & 23 & 8 & 11 & 42 \\
\hline NA & 8 & 0 & 3 & 11 \\
\hline \multicolumn{2}{|l|}{ EBV Latency profile ${ }^{\mathrm{a}}$} & 1 & & \\
\hline I & 1 & 1 & 0 & 2 \\
\hline II & 5 & 0 & 2 & 7 \\
\hline III & 1 & 0 & 1 & 2 \\
\hline NA & 4 & 1 & 3 & 8 \\
\hline \multicolumn{5}{|l|}{ Lytic EBV replication } \\
\hline Present & 2 & & 1 & 3 \\
\hline Absent & 1 & 1 & 2 & 4 \\
\hline NA & 8 & 1 & 3 & 12 \\
\hline \multicolumn{5}{|c|}{ IgH/IgK rearrangement } \\
\hline M & 10 & 2 & 2 & 14 \\
\hline MP & 4 & 2 & 1 & 7 \\
\hline $\mathrm{P}$ & 2 & 4 & 8 & 14 \\
\hline NA & 26 & 2 & 9 & 37 \\
\hline \multicolumn{5}{|l|}{ TCR rearrangement } \\
\hline M & 3 & 0 & 0 & 3 \\
\hline MP & 0 & 1 & 1 & 2 \\
\hline $\mathrm{P}$ & 0 & 4 & 3 & 7 \\
\hline NA & 39 & 5 & 16 & 60 \\
\hline \multicolumn{5}{|c|}{ Initial therapy response } \\
\hline Complete response & 17 & 3 & 10 & 30 \\
\hline Partial response & 1 & 0 & 0 & 1 \\
\hline Stable disease & 1 & 1 & 0 & 2 \\
\hline Progressive disease & 10 & 0 & 2 & 12 \\
\hline NA & 13 & 6 & 8 & 27 \\
\hline
\end{tabular}

EBER ISH EBV RNA in situ hybridization, EBV Epstein-Barr virus, $M$ monoclonal, $M P$ monoclonal in polyclonal background, $N A$ not available, $P$ polyclonal

a 0: LMP1-/EBNA2-/EBNA1-; I, LMP1-/EBNA2-/EBNA1+; II, LMP1+/EBNA2-; and III, LMP1+/EBNA2+

lymphoma, and classical Hodgkin lymphoma based on the classification used for post-transplant lymphoproliferative disorders. Twelve cases $(12 / 72,19 \%)$ were included in the non-destructive lymphoproliferative disorder category: 11 follicular hyperplasias and one EBV $+/ \mathrm{HHV} 8+$ germinotropic lymphoproliferation. Eight cases $(8 / 72,11 \%)$ were classified as polymorphic lymphoproliferative disorders: seven polymorphic lymphoproliferative processes as described for post-transplant lymphoproliferative disorders and one lymphomatoid granulomatosis. In the non-Hodgkin lymphoma category we included $44 / 72$ cases (61\%): twenty-two diffuse large B-cell lymphomas, four extranodal marginal zone lymphomas of mucosa-associated lymphoid tissues, four hepatosplenic T-cell lymphomas, two Burkitt lymphomas, two primary diffuse large B-cell lymphomas of the central nervous system, two chronic lymphocytic leukamias/small lymphocytic lymphomas, one primary diffuse large B-cell lymphoma of testes, one extranodal NK/T-cell lymphoma (nasal type), one plasmablastic lymphoma, one extramedullary plasmacytoma, one T-cell acute lymphoblastic leukemia-lymphoma, one follicular lymphoma (grade 2), and two B-cell non-Hodgkin lymphomas that could not be further diagnosed based on the available tissue. Six cases $(6 / 72,8 \%)$ were grouped in the Hodgkin lymphoma category: three mixed cellularity classical Hodgkin lymphomas, two nodular sclerosis classical Hodgkin lymphomas, and one lymphocyte-rich classical Hodgkin lymphoma. The remaining two cases could not be included in a specific category with enough certainty and were considered unclassifiable. Sixty-seven cases could be classified as therapy related according to the 2017 revised WHO classification of tumors of haematopoietic and lymphoid tissues [5]. The other at present not officially recognized categories (two chronic lymphocytic leukemias/small lymphocytic lymphomas, one follicular lymphoma, one extramedullary plasmacytoma, and one T-acute lymphoblastic leukemialymphoma) were nevertheless included (Table 2; full list in supplementary table). There were significantly more nonHodgkin lymphomas compared with polymorphic and reactive/non-destructive lymphoproliferative disorders in the immunosuppressive group vs the immunomodulatory and the immunosuppressive + immunomodulatory groups $(88 \%(35 / 40)$ vs $43 \%(3 / 7)$ and $35 \%(6 / 17))$. The monomorphic lymphomas comprised 39/44 (89\%) B-cell, and 5/44 (11\%) T-cell lymphomas. 6/50 (12\%) were classical Hodgkin lymphomas. The most common lymphoma in the population was diffuse large B-cell lymphoma, not otherwise specified $(36 \%(26 / 72))$, of which $26 \%(6 / 23)$ were of germinal center B-cell and 74\% (17/23) were of nongerminal center B-cell origin as determined by the Hans algorithm [35]. In three cases there was insufficient biopsy material to do the required immunohistochemical stainings. Material for EBER ISH was available in 61 cases. In total $31 \%(19 / 61)$ of cases were EBV-positive. Comparisons of the proportions of EBV-driven cases with the autoimmune disease therapy based subgroups (Table 3 ) revealed no significant differences with $p$-values of 0.6969 (immunosuppressive vs immunomodulatory), 0.8336 (immunosuppressive vs immunomodulatory + immunosuppressive), and 0.6655 (immunomodulatory vs immunomodulatory + immunosuppressive). Within the lymphoproliferative disorder classes $10 \%(1 / 10)$ of non-destructive lymphoproliferative disorders, $75 \%(6 / 8)$ of polymorphic lymphoproliferative disorders, 20\% (1/5) of Hodgkin lymphomas, and 11/37 (30\%) non-Hodgkin lymphoma were EBER ISH+. EBV latency profile could be assessed in 11/ 
19 EBER + cases with predominantly latency type II (64\% (7/11)). ZEBRA staining for lytic viral replication could be done in seven cases and nuclear positivity was detected in only $3 / 7$ cases. In two patients, both from the immunosuppressive group, there were only rare positive cells (less than 5/HPF), while in one patient, under immunosuppressive + immunomodulatory therapy, one diffuse large B-cell lymphoma showed a higher number of positive cells (15-20 per HPF) [33]. The latter lymphoma developed approximately 8 years after diagnosis of the underlying autoimmune disorder and 19 months after combined immunomodulatory + immunosuppressive therapy was initiated.

\section{Survival and time-to-event analysis}

The difference in mean duration between underlying therapy groups was not significant (Fig. 1a). Cox-proportional hazard analysis did reveal a significantly shorter time to lymphoproliferative disorders development (Fig. 1b, c) for immunomodulatory, immunomodulatory + immunosuppressive, and immunomodulatory-all when compared with immunosuppressive patients. Kaplan-Meier analysis revealed a trend toward a decreased long-term survival for the immunosuppressive group (Fig. 1d). Other analyses comparing survival for EBV-positive vs EBV-negative cases, International Prognostic Index scoring scores and
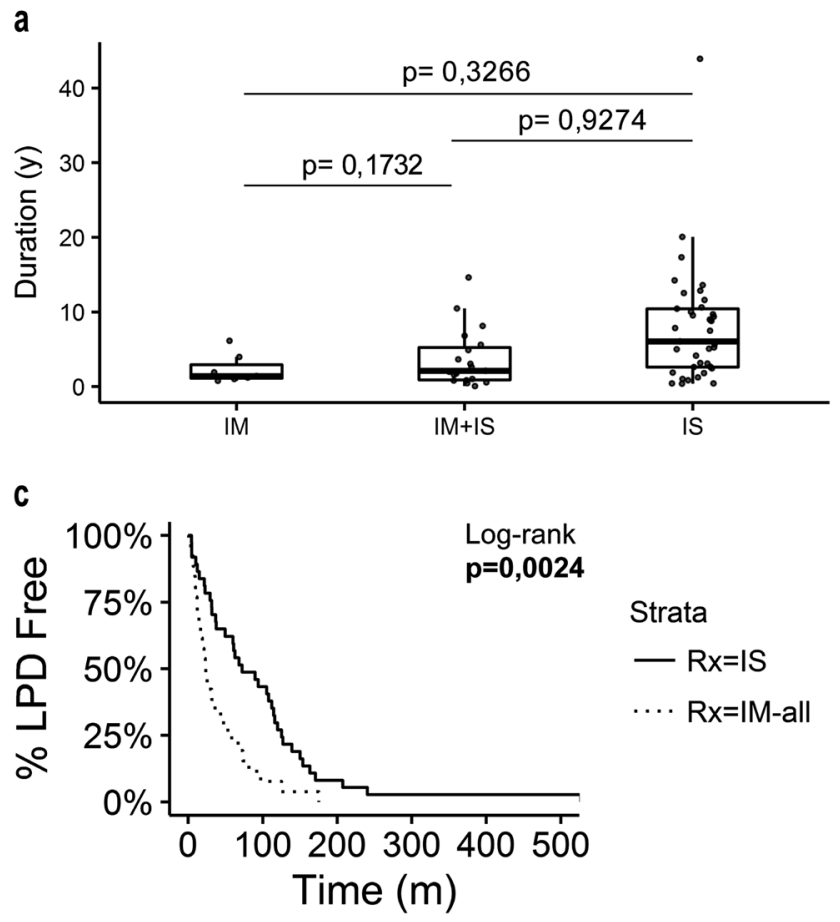

Fig. 1 a Boxplot of the underlying therapy duration until LPD diagnosis. b-c Cox-proportional hazard analysis of LPD free patients over time for the different underlying therapy groups (b) and IS vs IM-all (c). d Kaplan-Meier survival analysis for the different underlying
Ann-Arbor stages are available in the supplemental data but revealed no significant differences (figure S2).

\section{Illustrative case descriptions}

The following cases were selected because of their relation to immunosuppression/immunomodulation and represent the presence of the different histological subtypes (nondestructive, polymorphic and monomorphic lesions), as described in post-transplant lymphoproliferative disorders.

Case 1, nondestructive EBV-driven lymphoproliferative disorder: a 24-year-old woman with diagnosis of both Crohn's disease and systemic lupus erythematosus presented with a chronically enlarged fluorodeoxyglucose PET-positive right-inguinal lymph node in 02/2016. She had been taking azathioprine since 2 years earlier and Infliximab since June 2009. PET-scan further revealed enlarged lymph nodes in the axillary, inguinal, and iliac regions (Ann-Arbor stage III). Biopsy of an inguinal lymph node showed a reactive lymph node with intact architecture, but EBER ISH staining showed presence of intra-follicular EBV-positive cells and HHV8 immunohistochemistry was positive in germinal centers as well (Fig. 2a-c). This is not the normal lymph node compartment for latently positive memory B cells and this specific presentation has been reported as a germinotropic lymphoproliferation in HIV-

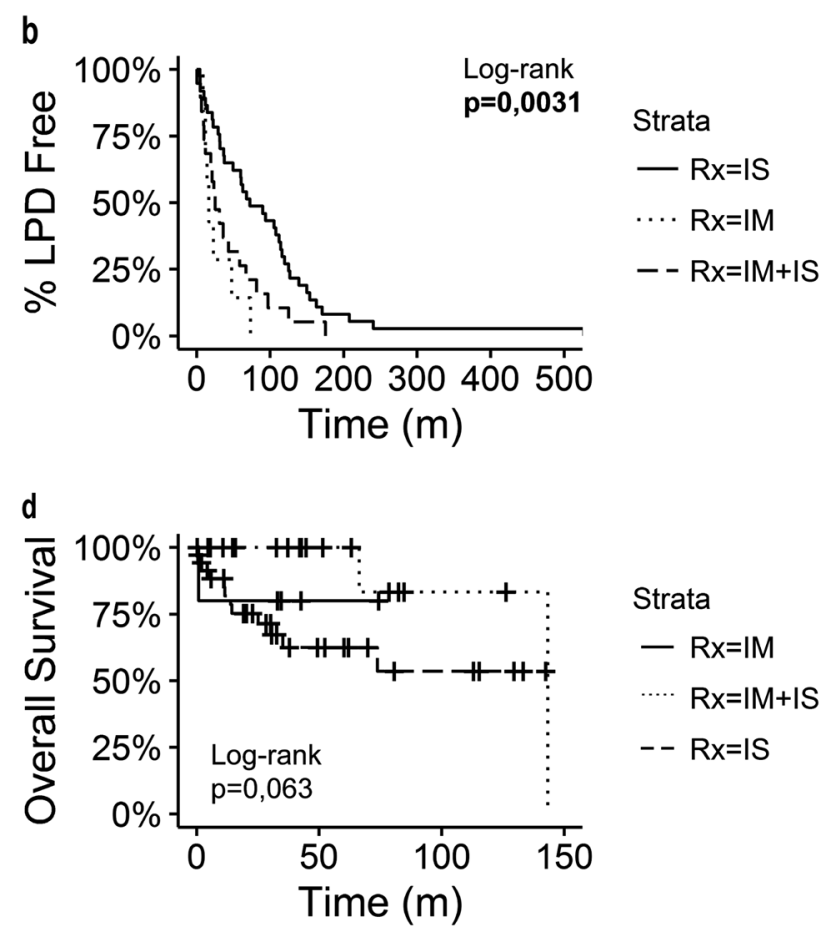

disease therapy groups. IM immunomodulatory, IS immunosuppressive, LPD lymphoproliferative disorder, $\mathrm{m}$ months, Rx Underlying disease therapy, $\mathrm{y}$ year 

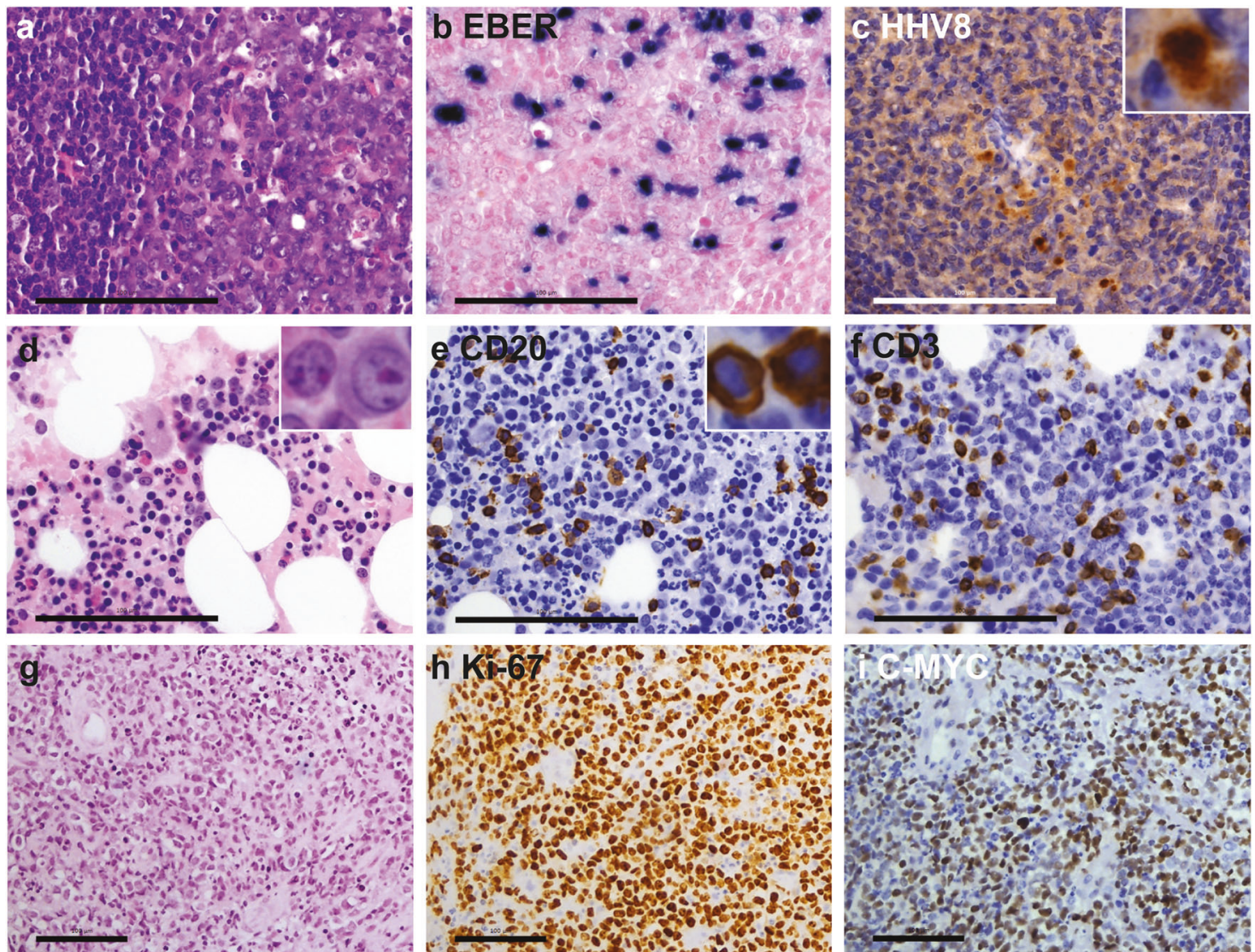

Fig. 2 Representative case images for case $1(\mathbf{a}-\mathbf{c})$, case $2(\mathbf{d}-\mathbf{f})$, and case 3 (g-i). All pictures were taken on a Leica DM2000 light microscope with a Leica DFC $7000 \mathrm{~T}$ camera; $\mathbf{a}-\mathbf{f} \times 20$ objective; $\mathbf{g}-\mathbf{i} \times 10$ objective; $\mathbf{c}-\mathbf{e}$ zoom with $\times 100$ oil objective; scale bar $=100$ $\mu \mathrm{m} ; \mathbf{d}-\mathbf{f}$ are pictures of a bone marrow biopsy, $\mathbf{a}-\mathbf{c}$ and $\mathbf{g}-\mathbf{i}$ are from lymph node biopsies; a, d, g: hematoxylin and eosin; b: EBV RNA in situ hybridization (EBER); c: HHV8 immunohistochemistry stain; e: CD20 IHC stain; f: CD3 IHC stain; h: Ki-67 IHC stain; i: C-MYC IHC stain. IHC immunohistochemical

Hodgkin/Reed-Sternberg like B cells (CD20+/CD30 $+/ \mathrm{EBV}-$ ) and increased $\mathrm{T}$ cell numbers both in the ileum and bone marrow (Fig. 2d-f). PCR for IGH rearrangement revealed a monoclonal pattern in a polyclonal background. The disease was staged Ann-Arbor IV with an International Prognostic Index score of 1. Immunomodulatory therapy (adalimumab) was halted and two cycles of anti-CD20 therapy (rituximab) were administered. The patient showed complete remission of all lesions and was lymphoproliferative disorder free at last follow-up 3.5 years later.

Case 3, Burkitt-like lymphoma (BL) with 11q aberration, a monomorphic lymphoproliferative disorder: a cervical lymph node core needle biopsy from a 25 -year-old male patient was performed in a peripheral hospital and sent to our center for a second opinion. The patient was treated with mercaptopurine for Crohn's disease for more than 10 years. Histopathological examination of the biopsy revealed diffuse sheets of medium-sized CD20+, CD10+, BCL6+ B-lymphocytes with a proliferation index of almost $100 \%$ (Ki-67/Mib-1 staining). Frequent apoptotic bodies and macrophages resulted in a starry sky pattern (Fig. $2 \mathrm{~g}-\mathrm{i}$ ). 
FISH examination showed no evidence for a $M Y C$-translocation $\mathrm{t}(8 \mathrm{q} 24 / M Y C)$ but three signals of the centromeric/ MLL/11q23 probe and loss of its telomeric region were detected. This is compatible with a Burkitt-like lymphoma with 11q aberration (ICD/0 9687/3) [39, 40]. This chromosomal abnormality is detected in $60 \%$ of EBV-negative Burkitt lymphomas and has been associated with immunocompromised patients [39]. Administration of 6mercaptopurine was stopped and treatment with rituximab combined with cyclophosphamide, doxorubicin, vincristine, and prednisone chemotherapy initiated, leading to a complete remission. At last follow-up, 14 months following lymphoproliferative disorder diagnosis, the patient was disease free.

\section{Discussion}

In this series we described the clinicopathological characteristics of 50 overt lymphomas (44 non-Hodgkin and six classical Hodgkin lymphomas) and 20 cases with nondestructive/reactive or polymorphic lymphoproliferative lesions associated with the suppression and/or modulation of the immune system by different drugs. Two cases could not be classified with certainty based on the available biopsy and were designated as "Unclassifiable." Each case was diagnosed according to the 2017 revised WHO classification of tumors of haematopoietic and lymphoid tissues [3] and subclassified similar to post-transplant lymphoproliferative disorders. Current treatment consists of stopping the immunosuppressive/immunomodulatory therapy when possible and is, depending of the type of lymphoproliferative disorders, followed by immune- and/or chemo-, and possible radiotherapy.

The increased risk for lymphoproliferative disorders in the post-transplant population [1,41-45] and case reports with a clear relation in time between therapy initiation and the development of lymphoproliferative disorders [46, 47] support the existence of a causative role between immunosuppressive/immunomodulatory therapy and lymphoproliferative disorders. This causative role of immunomodulatory/suppressive therapy in immunosuppressive/immunomodulatory agent-related lymphoproliferative disorders has been repeatedly suggested by case reports of lymphoproliferative disorders regression following discontinuation of methotrexate [48-52], anti-TNF $\alpha$ therapy [53-56] thiopurines [55], tacrolimus [57], and others [56]. A limitation in studying the effect of these drugs on lymphoproliferative disorder incidence is the still open question how long after cessation of therapy the immunosuppressive/immunomodulatory effects of these drugs linger. The 1-year cut-off used to classify cases into different therapy subgroups is based on similar cut-offs used in post-transplant lymphoproliferative disorder studies [4] but nevertheless, remains somewhat arbitrary. Another drawback is that patients in academical hospitals are often referred by other medical centers and parts of the earlier medical history are frequently lacking. As a result, the absence of errors in these records cannot be guaranteed. Furthermore, with only 72 patients some findings could have been missed due to an insufficient sample size. The five lymphoma cases that are currently not recognised as therapy related (two chronic lymphocytic leukamias/small lymphocytic lymphomas, one follicular lymphoma, one extramedullary plasmacytoma, and one T-acute lymphoblastic leukemia-lymphoma) were not excluded since they did develop in patients taking immunomodulatory/immunosuppressive medication. Notwithstanding these limitations this study is to the best of our knowledge one of the largest series of single-center "other immunomodulatory agent-related lymphoproliferative diseases" and provides more insight in the general characteristics of this disorder. The presence of the entire spectrum of lesions as exists in post-transplant lymphoproliferative disorders, which ranges from benign, nondestructive hyperplasia to monomorphic, overt malignancy, suggests a shared underlying lymphomagenesis mechanism for immunosuppressive and immunomodulatory therapy lymphoproliferative disorders similar to that in patients post-transplantation.

One difference is that the presence of EBV in our series of immunomodulatory/suppressive agent-related lymphoproliferative disorders is significantly lower than the series of post-transplant lymphoproliferative disorders (31 vs $66 \% ; p=1.829 \mathrm{e}-05$ ) [4]. This could either reflect a higher intensity of immune suppression in post-transplant lymphoproliferative disorders patients compared with immunomodulatory/suppressive agent-related lymphoproliferative disorder patients, or the presence of other pathogenic mechanisms related to the underlying autoimmune disorder, which might play a role. When comparing the subgroups, our observed incidence of EBV-association is lower than in some previously reported series of immunomodulatory/suppressive agent-related lymphoproliferative disorders $(82 \%)[9,10]$ but comparable to others $(38,41 \%)$ $[6,13]$. Differences in study design and focus make it difficult to accurately interpret these differences. The study of 17 cases by Hasserjian et al. [13], with a highly comparable design to our study, reported $41 \%$ (7/17) EBV-association with $28 \%(8 / 29)$ in the patient group meeting the same selection criteria. In that study, however, no cases receiving "only immunosuppressive therapy" were included. Differences in the proportion of EBV-related lymphoproliferative disorders between studies could be caused by the underlying study population with more EBV-driven lymphoproliferative disorders in Asia/Japan compared with Europe [58, 59]. Nevertheless, the proportion of EBV-positive 
immunomodulatory/suppressive agent-related lymphoproliferative disorder cases in our series is higher than in the immunocompetent population [59] $( \pm 2 \%)$. It seems likely that this reflects the impact of immunosuppression, as in patients with post-transplant lymphoproliferative disorders, but to a less pronounced extent.

Another significant difference with post-transplant lymphoproliferative disorders patients is the proportion of lymphoproliferative disorders developing less than 1 year after immunosuppressive/immunomodulatory therapy initiation. The incidence in the first year was lower here compared with the post-transplant lymphoproliferative disorders from our center [4] (18 vs 41\%; $p=0.04151$ ). Induction therapy given post-transplantation could explain this. The difference did drop below significance when hematopoietic stem cell transplant patients were excluded (18 vs $24 \% ; p=0.5434$ ), which is in accordance with the previously reported finding that post hematopoietic stem cell transplantation lymphoproliferative disorders arise earlier after transpantation [33]. The observed gender difference between nonmalignant lymphoproliferations (7/7 men) and lymphoma (8/11 women) reported by Hasserjian et al. was not confirmed in the patients included in this study. Even more, the trend $(p=0.05621)$ points toward the opposite with a majority of malignant lymphomas in male patients (11/14). Differences in selection criteria make comparisons with other studies less informative. Published studies almost always focus only on specific drugs [6, 53], autoimmune diseases [60, 61], malignancies [10-12], and/ or specific combinations [62], such as methotrexateassociated lymphoproliferative disorders in rheumatoid arthritis patients [7, 8,63-65].

When dividing the cases in groups according to the therapy administered in the year preceding lymphoproliferative disorder diagnosis, it becomes possible to assess differences that are potentially caused by specific therapies. Because of highly varying therapeutic regimens, we divided the cases in three groups: immunosuppressive, immunomodulatory, and immunosuppressive + immunomodulatory for immunosuppressive agent, immunomodulatory agent, and drug combination treated patients, respectively. We observed differences in time to lymphoproliferative disorder development with a shorter time for the immunomodulatory and immunomodulatory + immunosuppressive groups. The proportion of malignant cases in patients receiving immunomodulatory therapy was, however, also significantly lower when compared with the immunosuppressive treated cases. Differences in follow-up could explain the earlier detection with also more "pre-malignant" lesions. Possible explanations for differences in follow-up are increased awareness among clinicians, leading to a lower threshold for biopsy taking in patients receiving chronic immunomodulatory therapy or the fact that immunomodulatory therapy consists in the vast majority out of monoclonal antibodies for which the administration requires a regular visit to the clinic. Another explanation could be the age difference in the therapy subgroups, with the immunosuppressive group being significantly older. Historically immunosuppression was the only available therapy and immunomodulatory therapy was not started unless there was insufficient disease control. Therefore, older patients with controlled disease are often treated with immunosuppressive therapy only and we expect more malignancy overall in an older population. Importantly, a similar difference in the proportions of malignant cases in different therapy groups (immunomodulatory vs immunosuppressive + immunomodulatory) could be found in the case series published by Hasserjian et al. [13] $(p=0.04214)$. This correlates with the observed trend in adverse survival, higher Ann-Arbor stage and the significantly higher measured International Prognostic Index score in the immunosuppressive group compared with the other groups in this study. A notable finding is that all patients described by Hasserjian et al. with polymorphic and nondestructive lesions received only immunomodulatory therapy and not immunosuppressive + immunomodulatory, which is in line with the results described here.

A correlation between Hepatosplenic T-cell lymphoma and both IBD and thiopurine treatment has been reported in literature [66-70]. Three out of four hepatosplenic T-cell lymphomas cases in our series occurred in patients with IBD ( $2 X$ morbus Crohn, $1 \mathrm{X}$ ulcerative colitis). Two of these patients took azathioprine and the third had switched from azathioprine to methotrexate approximately 3 years prior to diagnosis. The fourth case was a rheumatoid arthritis patient receiving hydroxychloroquine. Due to the great differences in the applied therapeutic regimens for specific patients correlations between individual drugs and lymphoproliferative disorders are difficult to assess in a group of this size. In our series no other clear associations between therapy type, underlying autoimmune disease and specific lymphoma type are discovered although the fact that diffuse large B-cell lymphoma is the most frequent lymphoma is consistent with previous publications [71]. We encourage other groups to report series of immunomodulatory/suppressive agent-related lymphoproliferative disorders to further investigate possible correlations.

We analyzed the largest retrospective clinicopathological study so far on "other immunomodulatory agent-related lymphoproliferative diseases" with the aim to further characterize this rare entity. Since the continuous expansion of both indications for immunosuppressive and immunomodulatory therapy and the increased number of available therapeutics, we warrant an increased awareness for the occurrence of immunomodulatory/suppressive agent-related -lymphoproliferative disorders in the years to come. Clinicians need to be aware that these lymphoproliferative 
disorders exist and be alert during follow-up since these lymphoproliferative disorders arise both early after therapy initiation and many years later. When a lymphoproliferative disorder is suspected, histopathological confirmation and correct classification is necessary to guide therapy. EBVdriven lymphoproliferative disorders are less frequent than in post-transplant lymphoproliferative disorders, nevertheless EBV ISH should be a part of routine pathological diagnostics.

We observed occurrence of immunomodulatory agenttreated lymphoproliferative disorders earlier after therapy onset compared with immunosuppressive treated cases but with more nonmalignant lesions. Both overt malignant lymphomas and nonmalignant lesions as seen in posttransplant lymphoproliferative disorders can develop, so this could suggest that the earlier detection and subsequent stop of immunosuppressive/immunomodulatory therapy can prevent the development of overt lymphomas. Further study in larger series is, however, needed to address this. We encourage pathologists to classify these lesions according to the most recent WHO classification. Larger multicenter studies are required to identify biomarkers that can predict which patients are at risk and should be followed-up more closely in the long-term.

Acknowledgements We are grateful for the fruitful collaboration with Prof. Dr. R. Westhovens (Department of Rheumatology, UZ Leuven university hospital), Prof. Dr. G. Van Assche (Department of Gastro-enterology, UZ Leuven university hospital), Dr. K Segers (department of Pathology, AZ Turnhout), and Dr. F Ballaux (Department of Pathology, Imelda Hospital). This study was supported by the concerted action grant from the KU Leuven no. 3M040406 (T.T.) (http://www.kuleuven.be/) and the research grant from FWO-Vlaanderen (G081411N to G.V. and T.T.). T.T. holds a Mandate for Fundamental and Translational Research from the "Stichting tegen Kanker" (2014-083). D.D. holds a Mandate for Clinical Research from the University Hospitals Leuven and from "Kom op tegen Kanker" and is a cofounder of "Stefanie's Rozen Fonds," "Fonds Tom Debackere voor lymfoomonderzoek" and "Fonds Jos en Mieke Vandevordt-Gaul voor de pathogenese van zeldzame lymfomen." G.V. holds the International Roche Chair in hematology and is a founder of the "Fonds Tom Debackere voor lymfoomonderzoek" and "Fonds Jos en Mieke Vandevordt-Gaul voor de pathogenese van zeldzame lymfomen" and cofounder of "Stefanie's Rozen fonds." L. $\mathrm{M}$. is a PhD student, financially supported by KU Leuven, Department of Imaging and Pathology, "Stefanie's Rozen fonds" and the "Fonds Tom Debackere." CB is a PhD student, financially supported by KU Leuven, Department of Oncology, grant by "Kom op tegen Kanker (Stand up to Cancer), the Flemish cancer society."

\section{Compliance with ethical standards}

Conflict of interest The authors declare that they have no conflict of interest.

\section{References}

1. Dierickx D, Habermann TM. Post-transplantation lymphoproliferative disorders in adults. N Engl J Med. 2018;378:549-62.

2. Morscio J, Tousseyn T. Recent insights in the pathogenesis of post-transplantation lymphoproliferative disorders. World $\mathrm{J}$ Transplant. 2016;6:505-16.

3. Swerdlow SH, Webber SA, Chadburn A, Ferry JA. Posttransplant lymphoproliferative diorders. In: Swerdlow $\mathrm{SH}$, Campo E, Harris NL, Jaffe ES, Pileri SA, Stein H(eds.) WHO classification of tumours of haematopoietic and lymphoid tissuesRevised 4th ed France, Lyon: IARC; 2017. p. 453-62.

4. Dierickx D, Tousseyn T, Sagaert X, et al. Single-center analysis of biopsy-confirmed posttransplant lymphoproliferative disorder: incidence, clinicopathological characteristics and prognostic factors. Leuk Lymphoma. 2013;54:2433-40.

5. Gaulard P, Swerdlow SH, Harris NL, Sundström C, Jaffe ES. Other iatrogenic immunodeficiency associated lymphoproliferative disorders. In: Swerdlow SH, Campo E, Harris NL, Jaffe ES, Pileri SA, Stein H, et al.. editors.. WHO classification of tumours of haematopoietic and lymphoid tissues. Revised 4th ed. France, Lyon: IARC; 2017. p. 462-4.

6. Inui Y, Matsuoka H, Yakushijin K, et al. Methotrexate-associated lymphoproliferative disorders: management by watchful waiting and observation of early lymphocyte recovery after methotrexate withdrawal. Leuk Lymphoma. 2015;56:3045-51.

7. Hoshida Y, Xu JX, Fujita S, et al. Lymphoproliferative disorders in rheumatoid arthritis: clinicopathological analysis of 76 cases in relation to methotrexate medication. J Rheumatol. 2007;34:322-31.

8. Tokuhira M, Watanabe R, Nemoto T, et al. Clinicopathological analyses in patients with other iatrogenic immunodeficiencyassociated lymphoproliferative diseases and rheumatoid arthritis. Leuk Lymphoma. 2012;53:616-23.

9. Gion Y, Iwaki N, Takata K, et al. Clinicopathological analysis of methotrexate-associated lymphoproliferative disorders: comparison of diffuse large B-cell lymphoma and classical Hodgkin lymphoma types. Cancer Sci. 2017;108:1271-80.

10. Loo EY, Medeiros LJ, Aladily TN, et al. Classical Hodgkin lymphoma arising in the setting of iatrogenic immunodeficiency. Am J Surg Pathol. 2013;37:1290-7.

11. Miranda RN, Loo E, Medeiros LJ. Iatrogenic immunodeficiencyassociated classical hodgkin lymphoma: clinicopathologic features of 54 cases reported in the literature. Am J Surg Pathol. 2013;37:1895-7.

12. Hollander P, Rostgaard K, Smedby KE, et al. Autoimmune and atopic disorders and risk of classical hodgkin lymphoma. Am J Epidemiol. 2015;182:624-32.

13. Hasserjian RP, Chen S, Perkins SL, et al. Immunomodulator agent-related lymphoproliferative disorders. Mod Pathol. 2009;22:1532-40.

14. Kovarik J. From immunosuppression to immunomodulation: current principles and future strategies. Pathobiology. 2013;80:275-81.

15. Baecklund E, Iliadou A, Askling J, et al. Association of chronic inflammation, not its treatment, with increased lymphoma risk in rheumatoid arthritis. Arthritis Rheum. 2006;54:692-701.

16. Weyand CM, Goronzy JJ, Kurtin PJ. Lymphoma in rheumatoid arthritis: an immune system set up for failure. Arthritis Rheum. 2006;54:685-9.

17. Wong AK, Kerkoutian S, Said J, et al. Risk of lymphoma in patients receiving antitumor necrosis factor therapy: a metaanalysis of published randomized controlled studies. Clin Rheumatol. 2012;31:631-6. 
18. Connell WR, Kamm MA, Ritchie JK, et al. Long-term neoplasia risk after azathioprine treatment in inflammatory bowel disease. Lancet. 1994;343:1249-52.

19. Farrell RJ, Ang Y, Kileen P, et al. Increased incidence of nonHodgkin's lymphoma in inflammatory bowel disease patients on immunosuppressive therapy but overall risk is low. Gut. 2000;47:514-9.

20. Lewis JD, Bilker WB, Brensinger C, et al. Inflammatory bowel disease is not associated with an increased risk of lymphoma. Gastroenterology. 2001;121:1080-7.

21. Dayharsh GA, Loftus EV, Sandborn WJ, et al. Epstein-Barr viruspositive lymphoma in patients with inflammatory bowel disease treated with azathioprine or 6-mercaptopurine. Gastroenterology. 2002;122:72-77.

22. Warman JI, Korelitz BI, Fleisher MR, et al. Cumulative experience with short- and long-term toxicity to 6-mercaptopurine in the treatment of Crohn's disease and ulcerative colitis. J Clin Gastroenterol. 2003;37:220-5.

23. Askling J, Brandt L, Lapidus A, et al. Risk of haematopoietic cancer in patients with inflammatory bowel disease. Gut. 2005;54:617-22.

24. Chiorean MV, Pokhrel B, Adabala J, et al. Incidence and risk factors for lymphoma in a single-center inflammatory bowel disease population. Dig Dis Sci. 2011;56:1489-95.

25. Herrinton LJ, Liu L, Weng X, et al. Role of thiopurine and antiTNF therapy in lymphoma in inflammatory bowel disease. Am J Gastroenterol. 2011;106:2146-53.

26. Masunaga Y, Ohno K, Ogawa R, et al. Meta-analysis of risk of malignancy with immunosuppressive drugs in inflammatory bowel disease. Ann Pharmacother. 2007;41:21-28.

27. Kandiel A, Fraser A, Korelitz B, et al. Increased risk of lymphoma among inflammatory bowel disease patients treated with azathioprine and 6-mercaptopurine. Gut. 2005;54:1121-5.

28. Wolfe F, Michaud K. The effect of methotrexate and anti-tumor necrosis factor therapy on the risk of lymphoma in rheumatoid arthritis in 19,562 patients during 89,710 person-years of observation. Arthritis Rheum. 2007;56:1433-9.

29. Leombruno JP, Einarson TR, Keystone EC. The safety of antitumour necrosis factor treatments in rheumatoid arthritis: meta and exposure-adjusted pooled analyses of serious adverse events. Ann Rheum Dis. 2009;68:1136-45.

30. Mariette X, Matucci-Cerinic M, Pavelka K, et al. Malignancies associated with tumour necrosis factor inhibitors in registries and prospective observational studies: a systematic review and metaanalysis. Ann Rheum Dis. 2011;70:1895-904.

31. Lopez-Olivo MA, Tayar JH, Martinez-Lopez JA, et al. Risk of malignancies in patients with rheumatoid arthritis treated with biologic therapy: a meta-analysis. JAMA. 2012;308:898-908.

32. Morscio J, Dierickx D, Ferreiro JF, et al. Gene expression profiling reveals clear differences between EBV-positive and EBVnegative posttransplant lymphoproliferative disorders. Am J Transplant. 2013;13:1305-16.

33. Morscio J, Finalet Ferreiro J, Vander Borght S, et al. Identification of distinct subgroups of EBV-positive post-transplant diffuse large B-cell lymphoma. Mod Pathol. 2017;30:370-81.

34. van Dongen JJM, Langerak AW, Brüggemann M, et al. Design and standardization of PCR primers and protocols for detection of clonal immunoglobulin and T-cell receptor gene recombinations in suspect lymphoproliferations: Report of the BIOMED-2 Concerted Action BMH4-CT98-3936. Leukemia. 2003;17:2257-317.

35. Hans CP, Weisenburger DD, Greiner TC, et al. Confirmation of the molecular classification of diffuse large B-cell lymphoma by immunohistochemistry using a tissue microarray. Neoplasia. 2004;103:275-82.

36. Gonzalez-Farre B, Martinez D, Lopez-Guerra M, et al. HHV8related lymphoid proliferations: a broad spectrum of lesions from reactive lymphoid hyperplasia to overt lymphoma. Mod Pathol. 2017;30:745-60.

37. Du M-Q, Diss TC, Liu H, et al. KSHV- and EBV-associated germinotropic lymphoproliferative disorder. Blood. 2002; $100: 3415-8$.

38. Said J, Isaacson PG, Campo E, Harris NL. HHV8-associated lymphoproliferative disorders. In: Swerdlow SH, Campo E, Harris NL, Jaffe ES, Pileri SA, Stein H, et al.. editors.. WHO classification of tumours of haematopoietic and lymphoid tissues. Revised 4th ed. France, Lyon: IARC; 2017. p. 325-9.

39. Ferreiro JF, Morscio J, Dierickx D, et al. Post-transplant molecularly defined Burkitt lymphomas are frequently MYC-negative and characterized by the 11q-gain/loss pattern. Haematologica. 2015;100:e275-e279.

40. Leoncini L, Campo E, Stein H, Harris NL, Jaffe ES, Kluin PM. Burkitt-like lymphoma with $11 \mathrm{q}$ aberration. In: Swerdlow SH, Campo E, Harris NL, Jaffe ES, Pileri SA, Stein H, et al.. editors.. WHO classification of tumours of haematopoietic and lymphoid tissues. Revised 4th ed. France, Lyon: IARC; 2017. p. 334.

41. Dharnidharka VR, Sullivan EK, Stablein DM, et al. Risk factors for posttransplant lymphoproliferative disorder (PTLD) in pediatric kidney transplantation: a report of the North American Pediatric Renal Transplant Cooperative Study (NAPRTCS). Transplantation. 2001;71:1065-8.

42. Younes BS, McDiarmid SV, Martin MG, et al. The effect of immunosuppression on posttransplant lymphoproliferative disease in pediatric liver transplant patients. Transplantation. 2000;70:94-99.

43. Opelz G, Henderson R. Incidence of non-hodgkin lymphoma in kidney and heart transplant recipients. Lancet. 1993;342:1514-6.

44. Opelz G, Döhler B. Lymphomas after solid organ transplantation: a collaborative transplant study report. Am J Transplant. 2004;4:222-30.

45. Caillard S, Dharnidharka V, Agodoa L, et al. Posttransplant lymphoproliferative disorders after renal transplantation in the United States in era of modern immunosuppression. Transplantation. 2005;80:1233-43.

46. Adams AE, Zwicker J, Curiel C, et al. Aggressive cutaneous Tcell lymphomas after TNF $\alpha$ blockade. J Am Acad Dermatol. 2004;51:660-2.

47. Deng A, Harvey V, Sina B, et al. Interstitial granulomatous dermatitis associated with the use of tumor necrosis factor alpha inhibitors. Arch Dermatol. 2006;142:198-202.

48. Kamel OW, van de Rijn M, Weiss LM, et al. Brief report: reversible lymphomas associated with Epstein-Barr virus occurring during methotrexate therapy for rheumatoid arthritis and dermatomyositis. N Engl J Med. 1993;328:1317-21.

49. Rizzi R, Curci P, Delia M, et al. Spontaneous remission of 'methotrexate-associated lymphoproliferative disorders' after discontinuation of immunosuppressive treatment for autoimmune disease. Review of the literature. Med Oncol. 2009;26:1-9.

50. Miyazaki T, Fujimaki K, Shirasugi Y, et al. Remission of lymphoma after withdrawal of methotrexate in rheumatoid arthritis: relationship with type of latent Epstein-Barr virus infection. Am J Hematol. 2007;82:1106-9.

51. Horie N, Kawano R, Kaneko T, et al. Methotrexate-related lymphoproliferative disorder arising in the gingiva of a patient with rheumatoid arthritis. Aust Dent J. 2015;60:408-11.

52. Kawahara A, Tsukada J, Yamaguchi T, et al. Reversible methotrexate-associated lymphoma of the liver in rheumatoid arthritis: a unique case of primary hepatic lymphoma. Biomark Res. 2015;3:10.

53. Brown SL, Greene MH, Gershon SK, et al. Tumor necrosis factor antagonist therapy and lymphoma development: twenty-six cases reported to the Food and Drug Administration. Arthritis Rheum. 2002;46:3151-8. 
54. Park S-H, Kim C-G, Kim J-Y, et al. Spontaneous regression of EBV-associated diffuse lymphoproliferative disease in a patient with rheumatoid arthritis after discontinuation of etanercept treatment. Rheumatol Int. 2008;28:475-7.

55. Alobaid A, Torlakovic E, Kongkham P. Primary central nervous system immunomodulatory therapy-induced lymphoproliferative disorder in a patient with ulcerative colitis: a case report and review of the literature. World Neurosurg. 2015;84:2074. e15-2074.e19.

56. Hurley MY, George MN, Leonardi CL, et al. A transient benign lymph node-based proliferation of T-cells simulating nonHodgkin lymphoma in a patient with psoriasis treated with tumor necrosis factor alpha and CD11a antagonists. Diagn Pathol. 2008;3:13.

57. Sekiguchi Y, Shimada A, Imai H, et al. Epstein-Barr virus-negative, CD5-positive diffuse large B-cell lymphoma developing after treatment with oral tacrolimus for mixed connective tissue disease: a case report and review of the literature. J Clin Exp Hematop. 2012;52:211-8.

58. Oyama T, Yamamoto K, Asano N, et al. Age-related EBV-associated B-cell lymphoproliferative disorders constitute a distinct clinicopathologic group: a study of 96 patients. Clin Cancer Res. 2007; 13:5124-32.

59. Hofscheier A, Ponciano A, Bonzheim I, et al. Geographic variation in the prevalence of Epstein-Barr virus-positive diffuse large B-cell lymphoma of the elderly: a comparative analysis of a Mexican and a German population. Mod Pathol. 2011;24:1046-54.

60. Mercer LK, Davies R, Galloway JB, et al. Risk of cancer in patients receiving non-biologic disease-modifying therapy for rheumatoid arthritis compared with the UK general population. Rheumatology. 2013;52:91-98.

61. Simon TA, Thompson A, Gandhi KK, et al. Incidence of malignancy in adult patients with rheumatoid arthritis: a meta-analysis. Arthritis Res Ther. 2015;17:212.

62. Dulai PS, Thompson KD, Blunt HB, et al. Risks of serious infection or lymphoma with anti-tumor necrosis factor therapy for pediatric inflammatory bowel disease: a systematic review. Clin Gastroenterol Hepatol. 2014;12:1443-51.
63. Mariette X, Cazals-Hatem D, Warszawki J, et al. Lymphomas in rheumatoid arthritis patients treated with methotrexate: a 3-year prospective study in France. Blood. 2002;99:3909-15.

64. Ichikawa A, Arakawa F, Kiyasu J, et al. Methotrexate/iatrogenic lymphoproliferative disorders in rheumatoid arthritis: histology, Epstein-Barr virus, and clonality are important predictors of disease progression and regression. Eur J Haematol. 2013;91: 20-28.

65. Yamada K, Oshiro Y, Okamura S, et al. Clinicopathological characteristics and rituximab addition to cytotoxic therapies in patients with rheumatoid arthritis and methotrexate-associated large B lymphoproliferative disorders. Histopathology. 2015;67:70-80.

66. Thayu M, Markowitz JE, Mamula P, et al. Hepatosplenic T-cell lymphoma in an adolescent patient after immunomodulator and biologic therapy for Crohn disease. J Pediatr Gastroenterol Nutr. 2005;40:220-2.

67. Rosh JR, Gross T, Mamula P, et al. Hepatosplenic T-cell lymphoma in adolescents and young adults with Crohn's disease: A cautionary tale? Inflamm Bowel Dis. 2007;13:1024-30.

68. Mackey AC, Green L, Liang L-C, et al. Hepatosplenic T cell lymphoma associated with infliximab use in young patients treated for inflammatory bowel disease. J Pediatr Gastroenterol Nutr. 2007;44:265-7.

69. Kotlyar DS, Osterman MT, Diamond RH, et al. A systematic review of factors that contribute to hepatosplenic T-cell lymphoma in patients with inflammatory bowel disease. Clin Gastroenterol Hepatol. 2011;9:36-41.

70. van de Meeberg MM, Derikx LAAP, Sinnige HAM, et al. Hepatosplenic T-cell lymphoma in a 47-year-old Crohn's disease patient on thiopurine monotherapy. World J Gastroenterol. 2016;22:10465-70.

71. Baecklund E, Sundström C, Ekbom A, Catrina AI, Biberfeld P, Feltelius N, et al. Lymphoma subtypes in patients with rheumatoid arthritis: increased proportion of diffuse large B cell lymphoma. Arthritis Rheum. 2003;48:1543-50.

72. Hanauer SB. Review article: aminosalicylates in inflammatory bowel disease. Aliment Pharmacol Ther. 2004;20(Suppl 1): $60-65$.

\section{Affiliations}

\section{Lukas Marcelis $^{1} \cdot$ Charlien Berghen $^{2} \cdot$ Alexandra De Zutter $^{1} \cdot$ Pauline Biesemans ${ }^{1} \cdot$ Peter Vandenberghe $^{3}$. Gregor Verhoef $^{3,4} \cdot$ Olivier Gheysens $^{5} \cdot$ Xavier Sagaert $^{1,6} \cdot$ Daan Dierickx ${ }^{3} \cdot$ Thomas Tousseyn $^{1,6}$}

1 Department of Imaging and Pathology, Translational Cell and Tissue Research Lab, KU Leuven, Leuven, Belgium

2 Department of Radiation Oncology, UZ Leuven, University Hospitals, Leuven, Belgium

3 Department of Hematology, UZ Leuven, University Hospitals, Leuven, Belgium
4 Center of Human Genetics, KU Leuven, Leuven, Belgium

5 Department of Nuclear Medicine, UZ Leuven, University Hospitals, Leuven, Belgium

6 Department of Pathology, UZ Leuven, University Hospitals, Leuven, Belgium 